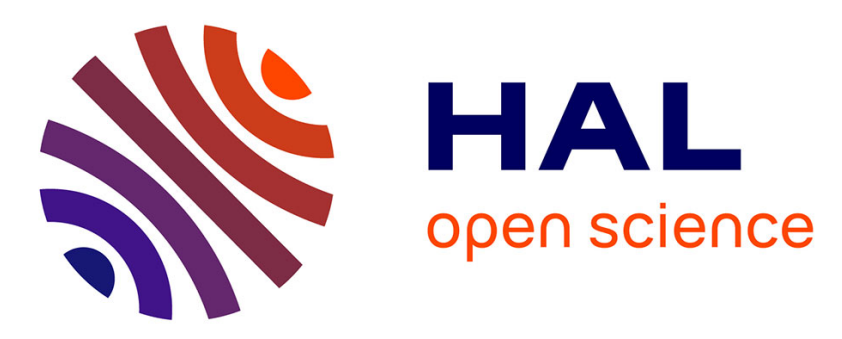

\title{
Human ACE2 peptide-mimics block SARS-CoV-2 pulmonary cells infection
}

Philippe Karoyan, Vincent Vieillard, Luis Gómez-Morales, Estelle Odile, Amélie Guihot, Charles-Edouard Luyt, Alexis Denis, Pascal Grondin, Olivier Lequin

\section{To cite this version:}

Philippe Karoyan, Vincent Vieillard, Luis Gómez-Morales, Estelle Odile, Amélie Guihot, et al.. Human ACE2 peptide-mimics block SARS-CoV-2 pulmonary cells infection. Communications Biology, 2021, 4 (1), pp.197. 10.1038/s42003-021-01736-8 . hal-03141346

\section{HAL Id: hal-03141346 https: / hal.sorbonne-universite.fr/hal-03141346}

Submitted on 15 Feb 2021

HAL is a multi-disciplinary open access archive for the deposit and dissemination of scientific research documents, whether they are published or not. The documents may come from teaching and research institutions in France or abroad, or from public or private research centers.
L'archive ouverte pluridisciplinaire HAL, est destinée au dépôt et à la diffusion de documents scientifiques de niveau recherche, publiés ou non, émanant des établissements d'enseignement et de recherche français ou étrangers, des laboratoires publics ou privés. 


\title{
communications biology
}

ARTICLE

https://doi.org/10.1038/s42003-021-01736-8

OPEN

\section{Human ACE2 peptide-mimics block SARS-CoV-2 pulmonary cells infection}

\author{
Philippe Karoyan (10) 1,2,3凶 , Vincent Vieillard ${ }^{4}$, Luis Gómez-Morales (10) 1,2, Estelle Odile ${ }^{1,2}$, Amélie Guihot ${ }^{5,6}$, \\ Charles-Edouard Luyt ${ }^{7}$, Alexis Denis (10 ${ }^{8}$, Pascal Grondin (1) ${ }^{8}$ \& Olivier Lequin ${ }^{1}$
}

In light of the recent accumulated knowledge on SARS-CoV-2 and its mode of human cells invasion, the binding of viral spike glycoprotein to human Angiotensin Converting Enzyme 2 (hACE2) receptor plays a central role in cell entry. We designed a series of peptides mimicking the $\mathrm{N}$-terminal helix of hACE2 protein which contains most of the contacting residues at the binding site, exhibiting a high helical folding propensity in aqueous solution. Our best peptide-mimics are able to block SARS-CoV-2 human pulmonary cell infection with an inhibitory concentration $\left(\mathrm{IC}_{50}\right)$ in the nanomolar range upon binding to the virus spike protein with high affinity. These first-in-class blocking peptide mimics represent powerful tools that might be used in prophylactic and therapeutic approaches to fight the coronavirus disease 2019 (COVID-19).

\footnotetext{
${ }^{1}$ Sorbonne Université, École Normale Supérieure, PSL University, CNRS, Laboratoire des Biomolécules, LBM, 75005 Paris, France. ${ }^{2}$ Sorbonne Université, École Normale Supérieure, PSL University, CNRS, Laboratoire des Biomolécules, LBM, Site OncoDesign, 25-27 Avenue du Québec, 91140 Villebon Sur Yvette, France. ${ }^{3} \chi$-Pharma, 25 avenue du Québec, 91140 Villebon Sur Yvette, France. ${ }^{4}$ Sorbonne Université, INSERM, CNRS, Centre d'Immunologie et des Maladies Infectieuses-Paris (CIMI-Paris), F-75013 Paris, France. ${ }^{5}$ Assistance Publique-Hôpitaux de Paris (AP-HP), Hôpital Pitié-Salpêtrière, Département d'Immunologie, F-75013 Paris, France. ${ }^{6}$ Sorbonne Université, Inserm U1135, Centre d'Immunologie et des Maladies Infectieuses, CIMI-Paris, F-75013 Paris, France. ${ }^{7}$ Assistance Publique-Hôpitaux de Paris (AP-HP), Hôpital Pitié-Salpêtrière, Service de Médecine Intensive Réanimation, Institut de Cardiologie,

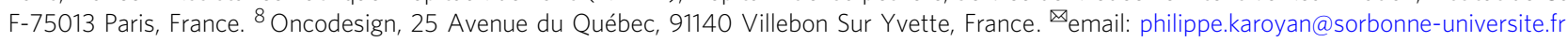


$\mathrm{T}$ he coronavirus disease 2019 (COVID-19), caused by the severe acute respiratory syndrome-coronavirus 2 (SARS$\mathrm{CoV}-2$ ) has emerged as a pandemic, claiming at the time of writing more than 1.3 million deaths and over 57 millions confirmed cases world-wide between December 2019 and November $2020^{1}$. Since the SARS-CoV-2 discovery ${ }^{2,3}$ and identification, the energy deployed by the scientific community has made it possible to generate an extraordinary wealth of information. However, to date, efficient therapeutics or drugs are lacking and prevention of the disease relies only on non-specific barrier measures ${ }^{4}$. Indeed, no specific drugs targeting the virus are available ${ }^{5}$ yet, many clinical trials have been engaged with SARS-CoV-2 non-specific treatments ${ }^{4}$. The structural and biochemical basis of the mechanism of infection has been investigated, highlighting that the virus cellsurface spike protein of SARS-CoV-2 targets human receptors ${ }^{6,7}$. Human angiotensin converting enzyme 2 (hACE2) and the cellular transmembrane protease serine 2 (TMPRSS2) have been identified as major actors of the virus entry into human cells ${ }^{8}$.

With the goal of preventing the SARS-CoV-2 from infecting human cells, blocking the interaction between hACE2 and the virus spike protein has been validated. Indeed, inhibition of SARS-CoV-2 infections in engineered human tissues using clinical-grade soluble ACE2 was recently demonstrated ${ }^{9}$. Likewise, an engineered stable mini-protein mimicking three helices of hACE2 to plug SARS-CoV-2 spikes ${ }^{10}$ was described, but its capacity to block viral infection was not demonstrated. Although several in silico designed peptides were proposed to prevent formation of the fusion core 11,12 , first attempts to design a peptide binder derived from hACE2 proved to be a difficult task, leading to mitigated results ${ }^{13}$.

Thus, starting from the published crystal structure of SARSCoV-2 spike receptor-binding domain (RBD) bound to hACE2 ${ }^{14}$, we designed peptide-mimics of the $N$-terminal hACE2 helix which interact with the spike protein. We report here the strategy implemented to optimize the design of our peptide mimics, their high helical folding propensity in water, their ability to block SARS-CoV-2 human pulmonary cell infection with an $\mathrm{IC}_{50}$ in the nanomolar range and their binding to spike RBD with strong affinity. We also demonstrated the non-toxicity of our mimics at concentrations 150 times higher than the $\mathrm{IC}_{50}$ on pulmonary cell lines.

\section{Results}

Design of peptides mimicking the helix $\mathrm{H} 1$ of hACE2. We first examined the complex between hACE2 and the surface spike protein of SARS-CoV-2 (PDB $6 \mathrm{~m} 0 \mathrm{j})^{14}$ in order to highlight the important contacts and some relevant characteristics of the interacting hACE2 sequence (Fig. 1).

Twenty residues from hACE2 were identified ${ }^{14}$ as to be in close contacts with the Spike protein, using a distance cut-off of $4 \AA$. These interactions occur mainly through the $N$-terminal $\alpha$-helix H1 of hACE2 (Fig. 1a). This a-helix (Fig. 1b), composed of 27 residues (from S19 to L45, Fig. 1c) contains 12 residues (highlighted in magenta in Fig. 1c) involved in hydrogen bonds, salt bridges, and van der Waals interactions (see Supplementary Table 1 for details) ${ }^{14}$.

A Clustal multiple sequence alignment of viruses isolated in China, United States and France was performed (See Supplementary Fig. 1). From these analyses, we observed that all the randomly selected sequences were $100 \%$ identical at least in the ACE2 interacting interface. This highlights a highly conserved sequence for the portion of spike interacting with the $\alpha$-helix H1 of hACE2, possibly because deleterious mutations at this interface would limit viral infectivity.

Our strategy was designing a peptide with a high helical folding propensity and retaining most of the binding affinity of hACE2 to the spike RBD of SARS-CoV-2, using natural amino acids ${ }^{15}$. Indeed, we preferred not to use complex chemical tools known to stabilize $\alpha$-helix ${ }^{16,17}$ in order to limit developability constraints. Our mimics were designed and optimized for binding, high helical content and low antigenicity, to avoid triggering a neutralizing immune response that would compromise the peptide therapeutic potential. A combination of the Agadir program $^{18,19}$, an algorithm developed to predict the helical content of peptides and the semi-empirical method reported by Kolaskar $^{20}$ to highlight the number of antigenic determinants, was iteratively used.

We observed that the $N$-terminal sequence of the $\mathbf{H 1}$ helix, composed of four residues $\left(\mathrm{S}_{19} \mathrm{TIE}_{22}\right)$, corresponds to a consensus $N$-capping box motif (SXXE) ${ }^{21}$. A capping box features reciprocal backbone-side-chain hydrogen-bonds favoring a helix initiation. Although this sequence does not adopt the H-bonded capping conformation in the crystal structure of the full protein, it could constitute a stabilizing element in the isolated helix when extracted from the protein context. These observations led us to a)

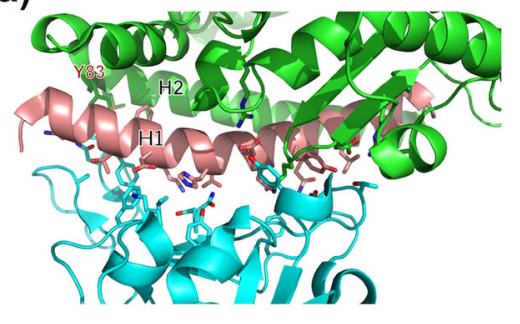

b)

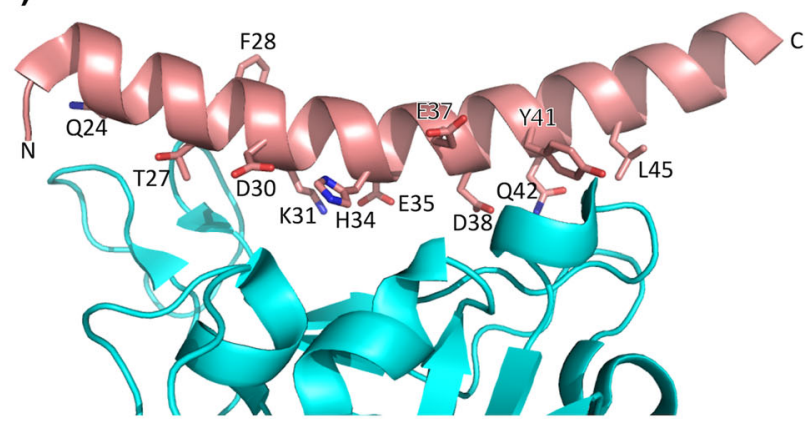

c) $-S_{19} T_{20} I_{21} E_{22} E_{23} Q_{24} A_{25} K_{26} T_{27} F_{28} L_{29} D_{30} K_{31} F_{32} N_{33} H_{34} E_{35} A_{36} E_{37} D_{38} L_{39} F_{40} Y_{41} Q_{42} S_{43} S_{44} L_{45}-$

Fig. 1 Structure of the complex between hACE2 and the spike protein of SARS-CoV-2 (pdb $\mathbf{6 m 0 j} \mathbf{m}^{\mathbf{1 4}}$. a Contact residues of the hACE2 / SARS-CoV-2 spike interface. hACE2 protein is colored in green, apart from $\mathrm{N}$-terminal helix $\mathrm{H} 1$ which is highlighted in salmon. SARS-CoV-2 spike protein is shown in cyan. b Residues of hACE2 H1 helix interacting with spike. c Sequence of hACE2 H1 helix showing the 12 interacting residues in magenta. Residue positions in green were considered as possible substitution sites for the helical peptide design. 
keep 14 residues from the native $\mathbf{H 1}$ helix of hACE2 as contact residues or putative stabilizing capping box. The 13 remaining residues that are not essential for the interaction were considered as possible sites for amino acid substitutions (Fig. 1c). We thus substituted non-essential positions by Ala and/or Leu residues which display higher helical folding propensities, and we calculated the peptide helical content after each substitution (Supplementary Table 2).

A peptide sequence optimization was then carried out to lower the antigenicity while keeping the helical propensity thanks to an iterative residue scanning and calculation of the helical content variation upon new substitutions (Supplementary Table 3). This strategy highlighted the influence of the residue N33 in the native sequence. Indeed, if the N33/L33 substitution systematically improved the helical content, it was always at the expense of antigenicity. Conversely, the L33/N33 substitution reduced the antigenicity at the expense of helical content. The solution was found by L33/M33 substitution which decreased the number of $\mathrm{AD}$.

The H1 helix of ACE2 adopts a kinked conformation in the crystal structure, leading to a distorted $\mathrm{CO} / \mathrm{HN}$ hydrogen bond network between H34/D38 and E35/L39 residues. Therefore, we considered introducing a proline, as this residue is known to induce local kinks or distortions in natural helices ${ }^{22,23}$. D38 was classed as a contact residue, while L39 side chain is not involved in any direct interaction. Consequently, L39 position was selected for substitution by proline (peptide P5, Table 1).

In order to increase the helical content to a maximum level, this iterative study was also applied to longer peptide sequences starting from the 29-residue native one, albeit at the expense of antigenicity. Diverse combinations of $N$ - and $C$-terminus capping groups were also considered (free extremities or $\mathrm{N}$-acetyl, $\mathrm{C}$ carboxamide groups).

Finally, we examined the possibility of promoting additional side chain contacts provided by ACE2 residues that do not belong to $\mathbf{H} \mathbf{1}$ helix. Y83 residue in $\mathbf{H} \mathbf{2}$ helix appeared as a good candidate as it lies very close in space to A25 in $\mathbf{H 1}$ helix (Fig. 1a). Molecular modeling was carried out on $\mathbf{H 1}$ helix analogs in which A25 was replaced by tyrosine or homotyrosine ( $h \mathrm{Tyr}$ ) residues (Supplementary Fig. 2). Calculations showed that $h$ Tyr residue was able to project the phenol ring in an adequate orientation to mimic Y83 position. Of note, $h$ Tyr is a natural amino acid ${ }^{24}$.

Three peptides were selected as controls in our optimization process, P1 (native sequence), P1scr (scrambled peptide from P1), and Ppen (described by Pentelute and colleagues in a longer biotinylated and pegylated construct and termed SBP1 as a putative spike binder ${ }^{13}$ ).

The results highlighting the progression in helical content and number of antigenic determinants are reported in Table 1 for the most relevant peptide mimics (see Supplementary Tables 2-4 for all the peptide-mimics that were designed and/or synthesized). These peptides were synthesized on a $5-\mathrm{mg}$ to $20-\mathrm{mg}$ scale from Fmoc-protected amino acids utilizing standard solid-phase peptide synthesis methods on rink amide resin (see Methods section).

The designed peptides highlight an excellent correlation between calculated and experimentally determined helical content by circular dichroism in aqueous media. The conformation of synthesized peptides in aqueous solution was investigated by circular dichroism (CD) spectroscopy ${ }^{25}$. Figure 2 shows the superimposed CD spectra of 12 peptides, including control ones, i.e., P1 (native sequence), P1scr (Scramble), and Ppen. The CD spectra of peptides P1 (native), P1scr (scrambled) are characteristic of a predominant random coil structure with a negative minimum near $200 \mathrm{~nm}$, as expected. Similarly, Ppen described as a helical peptide sequence ${ }^{13}$ also adopted a random coil conformation in solution. For all other peptides, the $\mathrm{CD}$ spectra exhibit a canonical $a$-helix signature, featuring a double minimum around $208 \mathrm{~nm}$ and $222 \mathrm{~nm}$, with the exception of the proline-containing P5 peptide. The deconvolution of the CD spectra using DichroWeb ${ }^{25}$ allowed us to estimate the helical population for each peptide, which is reported in Table 1. Overall, an excellent agreement was observed between the Agadircomputed values and the experimental helical population inferred from CD data. The native hACE2 $\mathbf{H 1}$ helix sequence (peptides P1, Ppen) has a weak propensity to fold into an $\alpha$-helix in aqueous solution (below 10\%). In contrast, the sequence optimization led to $\mathbf{H} \mathbf{1}$ analogs exhibiting a high helical propensity (between 50 and $80 \%$ ). The introduction of a proline residue in peptide P5 has a strong destabilizing effect on helical conformation $(17 \%)$ whereas Leu/hTyr substitution only led to a slight decrease of the helical content (P7 versus P6 and P10 versus P8).

Peptide mimics of hACE2 show high anti-infective efficacy and are devoid of cell toxicity. To determine whether our peptide mimics of hACE2 $\mathbf{H 1}$ helix block SARS-CoV-2 cell infection, antiviral assays ${ }^{26}$ were performed (Fig. 3) with a SARS-CoV-2 clinical isolate obtained from bronchoalveolar lavage (BAL) of a symptomatic infected patient (\#SARS-CoV-2/PSL2020) at PitiéSalpêtrière hospital, Paris (France) (see Methods section). We first measured the inhibition of viral replication in Vero-E6 cell cultures exposed to $10 \mu \mathrm{M}$ of the first set of peptide-mimics (P2 to P8, P1, P1scr, and Ppen being used as controls), for $48 \mathrm{~h}$ (Fig. 3a). These preliminary assays helped us to identify two peptide-mimics that stand out (P7 and P8) for their ability to block the viral infection, highlighting a potential role of $h$ Tyr. This observation helped us in the peptide-mimics structure optimization process. Two new peptides were designed, P9 and P10 incorporating the $h$ Tyr residue and evaluated with P8 for their ability to block viral infection on Vero-E6 cells through the measurement of infectious virus production and viral genome (Fig. 3b and Supplementary Fig. 3) ${ }^{27}$. These peptides proved to be devoid of toxicity in Vero-E6 (Fig. 3c). In order to get insight on their ability to block viral infection on human pulmonary cells, Calu-3 cell line (ATCC HTB55) was chosen. This pulmonary epithelial cell line is commonly used as a respiratory models in preclinical applications ${ }^{28}$ and SARS-CoV-2 has been shown to replicate efficiently in this cell line ${ }^{29}$.

We first observed a dose-dependent reduction in virus titer (Fig. 3d) and then, using ELISA assays we evaluated the average median inhibitory concentration $\left(\mathrm{IC}_{50}\right)$ on Calu-3 cells for $\mathbf{P 8}$, P9, and P10 to be $46 \mathrm{nM}, 53 \mathrm{nM}$, and $42 \mathrm{nM}$ respectively (Fig. 3e). Importantly, no cytotoxicity was observed in similarly treated uninfected culture cells at $10 \mu \mathrm{M}$, a concentration 150 times higher than the $\mathrm{IC}_{50}$ (Fig. 3f). Collectively, these data demonstrate the high antiviral potency of peptide analogs P8, P9, and P10.

The designed peptides bind to SARS-CoV-2 spike RBD with high affinity. Finally, the peptides that were able to block cell infection with an $\mathrm{IC}_{50}$ in the sub- $\mu \mathrm{M}$ range $(\mathbf{P 8}, \mathbf{P 9}$, and P10) were evaluated for their ability to bind to SARS-CoV-2 spike RBD (Fig. 4) using biolayer Interferometry (BLI) with an Octet RED96e system (FortéBio) ${ }^{30}$. hACE2 was used as a positive control (Fig. 4a).

Even though this technique presents some drawbacks ${ }^{31}$ offering narrow signal windows with low molecular weight analytes $^{32}$ such as peptides, it remained useful to identify and 
ARTICLE

COMMUNICATIONS BIOLOGY | https://doi.org/10.1038/s42003-021-01736-8

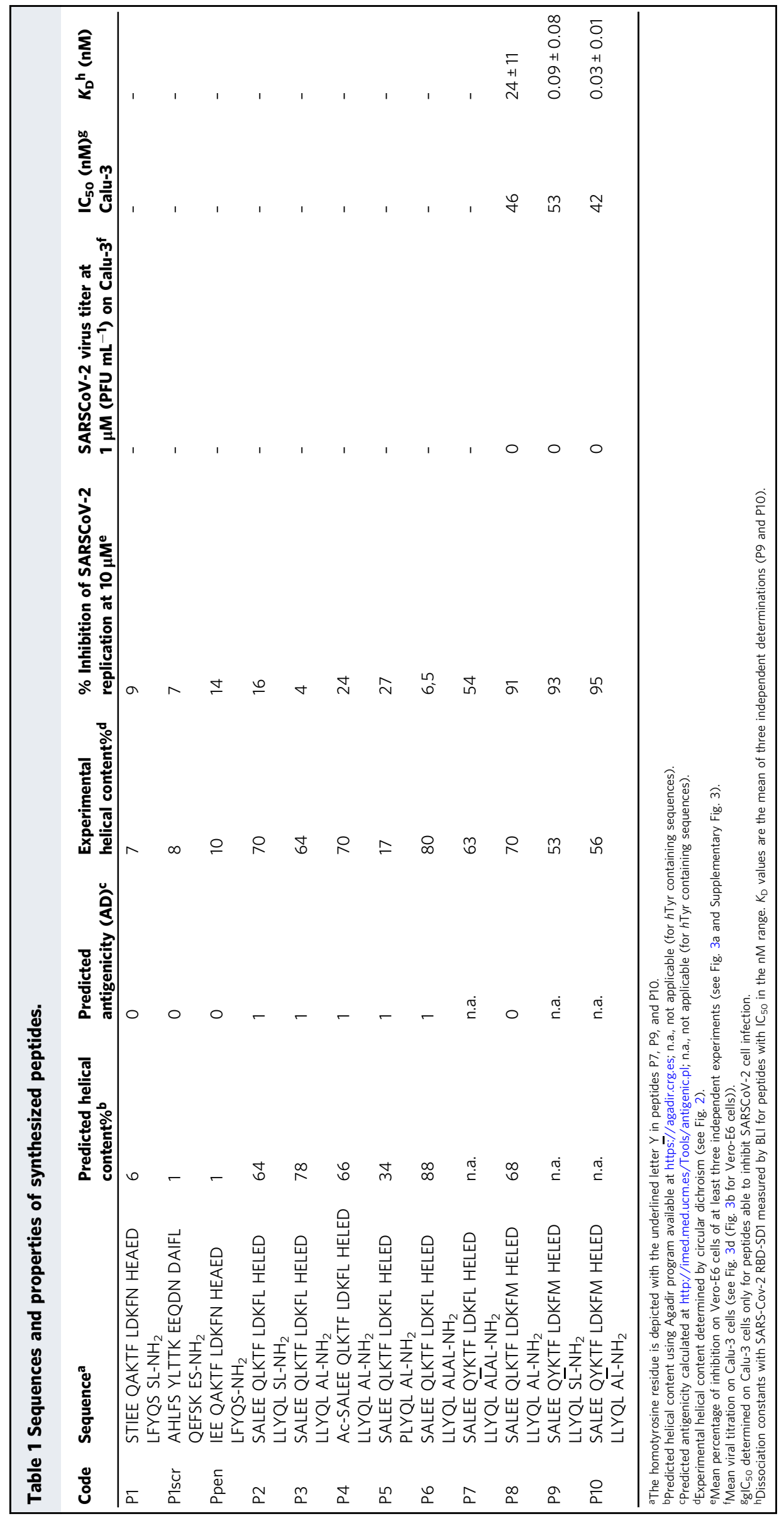

COMMUNICATIONS BIOLOGY | (2021)4:197| https://doi.org/10.1038/s42003-021-01736-8| www.nature.com/commsbio 


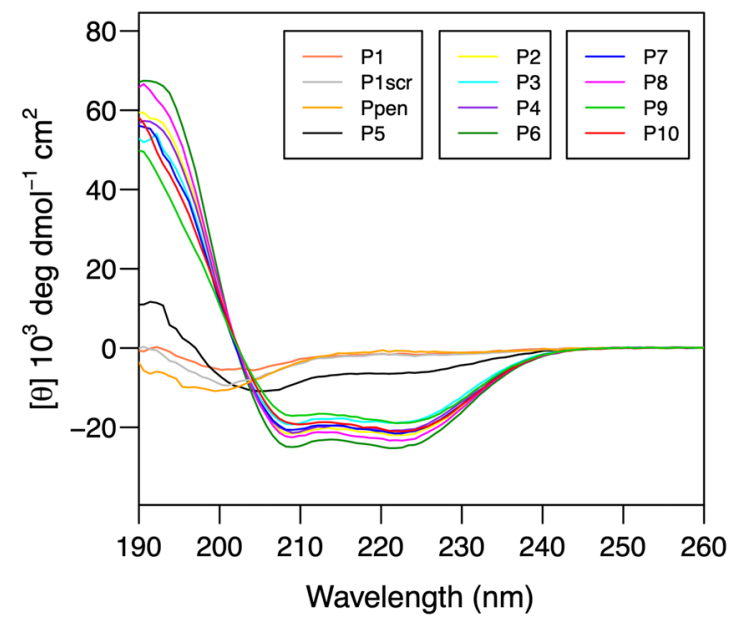

Fig. 2 Far-UV CD spectra of synthesized peptides. Samples of peptides P1-P10, P1Scr, and Ppen were prepared at a concentration of $60 \mu \mathrm{M}$ in $50 \mathrm{mM}$ sodium phosphate buffer at $\mathrm{pH}$ 7.4. CD measurements are reported as molar ellipticity per residue.

rank our binding mimics. In the conditions tested here, peptide P1 does not bind to RBD when using $100 \mathrm{nM}$ nor $10 \mu \mathrm{M}$ peptide solutions (Fig. 4b). For peptides P8, P9, and P10, multiple concentrations experiments were performed and dose-dependent associations were observed (Fig. 4c-e). Of note, only association rates could be quantified accurately, the dissociation ones being very slow, highlighting strong binding properties for these mimics (Supplementary Table 6).

\section{Discussion}

The current pandemic originated by SARS-CoV-2 causes an unprecedented health crisis. The medical world has found itself helpless in the face of this virus, having to deal with the absence of specific effective treatment. At the time of writing, preventive vaccines are not yet clinically approved, other antiviral drugs have been shown ineffective, and specific drugs addressing SARS-CoV2 targets are lacking ${ }^{4}$. Among all possible viral targets, the virus spike protein/hACE2 interaction has been validated and the design of compounds able to block this interaction upon binding to spike protein is a promising approach. However, developing a specific drug at a pandemic speed is a hard task especially in the design of a small molecule. Indeed, beyond the time required for the identification and validation of a lead compound after a library screening, followed by structure-activity relationship studies and clinical development, small molecule drugs are associated with a high attrition rate partly due to their off-target toxicity observed during pharmacological studies.

Peptides appear here as a possible solution for design and development at pandemic speed. Peptides are widely recognized as promising therapeutic agents for the treatment of various diseases such as cancer, and metabolic, infectious, or cardiovascular diseases ${ }^{33-35}$. To date, $\sim 70$ peptide drugs have reached the market and 150 are currently under clinical development ${ }^{33,36}$. Special advantages that peptides show over small drugs include their high versatility, target-specificity, lower toxicity, and ability to act on a wide variety of targets ${ }^{35}$ which are directly responsible for a greater success rate than small molecules (approval rate of around $20 \%$ versus $10 \%)^{36,37}$. The synthesis and the development of long therapeutic peptides (over 30 residues) are no longer a challenge, as highlighted by the success story of many GLP-1 analog $3^{8}$. Their possible antigenicity can be evaluated using prediction tools in the design 20 .
Of course, even for peptides, the development of a drug at a pandemic speed requires some considerations. Our aim was to design a peptide with reasonable helical folding propensity in water in order to mimic the H1 helix of hACE2 in the protein context, considering that this helical folding is a prerequisite to compete with hACE2 upon interaction with viral spike protein. The design was realized using only natural amino acids ${ }^{15}$ and avoided complex tools that are known and validated to stabilize $\alpha$-helix ${ }^{16}$. Stabilizing a-helical structure of medium-sized peptide sequences (up to 15 residues) using only natural amino acids is a hard but achievable task $^{39}$. Our choice was guided by the desire to build a simple peptide that can be upscaled quickly and easily, without technical constraints that may require laborious development. We also assumed that the use of mostly natural amino acids can facilitate the essential stages of the development of therapeutic tools in the event of success, particularly concerning pharmacokinetics, preclinical, and clinical toxicity aspects. We thought it would be a fair compromise between designing $\alpha$-helix peptides with optimized binding affinity and developing an effective tool within short deadlines while integrating the constraints of developability to move a prophylactic device and/or a therapeutic peptide drug quickly in the clinic.

Using a combination of validated methods, we improved the helical folding propensity of the native $\alpha$-helix extracted from the protein context. Thanks to leucine and alanine scanning (Table 1 and Supplementary Tables 2 and 3) a first set of peptides (P1 to P8) were designed and synthesized. These peptides demonstrated to have a high helical content (up to $80 \%$ for P6). However, increasing this helical content to a maximum level led to increasing mean hydrophobicity and a hydrophobic moment (Supplementary Table 4) that proved to be detrimental to solubility and efficacy. The substitution of a leucine residue by the homotyrosine residue led to the peptide analog $\mathbf{P 7}$ with a slight increase in solubility and a weak efficiency to block SARS-CoV-2 cell infection. In this first generation of peptides, the 27-residue P8 peptide appeared to be highly soluble with a high helical folding propensity (70\%), and an ability to block SARS-CoV-2 cell infection at $10 \mu \mathrm{M}$ on Vero-E6 cells (Fig. 3a).

In order to improve the potency of our peptides, we designed a second set of mimics combining the properties of peptides P7 and P8, i.e., P9 and P10. If the Leu/hTyr substitution led to a slight decrease in helicity, this was at the advantage of the mean hydrophobicity (Supplementary Table 4) also highlighted by lower HPLC retention times (Supplementary Table 5 and Supplementary Data 1). These peptides proved to be highly efficient in reducing SARS-CoV-2 viral titers (100\% efficacy at $1 \mu \mathrm{M})$ on pulmonary cells with an $\mathrm{IC}_{50}$ in the nanomolar range. This blocking property is related to their ability to bind to SARS-CoV-2 spike RBD with affinity estimated in the sub-nanomolar range (Table 1 and Supplementary Table 6). Finally, these peptides proved to be devoid of cell toxicity at 150 times the $\mathrm{IC}_{50}$ concentration (Fig. 3c, f) highlighting their therapeutic potential.

In conclusion, we demonstrated here the feasibility of designing hACE2 peptide-mimics with high helical folding propensity in water. The folding propensity promotes interaction with spike RBD and blocks SARS-CoV-2 pulmonary cell infection. Devoid of cell toxicity even at high doses, these mimics could be considered for prophylactic or therapeutic purposes upon adequate formulation. Targeting prophylaxis first might shorten the drug development time scale. Delivered through a medical device such as a nasal or oral spray or as a sublingual tablet, these peptides could be aimed at blocking the infectivity of the virus in a preventive manner. Their biodistribution would be limited to the upper airways (nasal and oral cavity) before they are degraded in the digestive tracks without toxic residues. Their therapeutic use might also be considered formulated in that case as an inhaler to reach pulmonary cells. 
a)

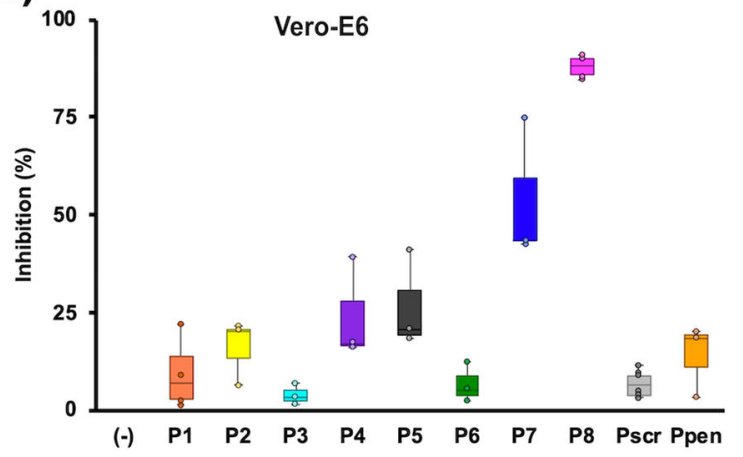

b)

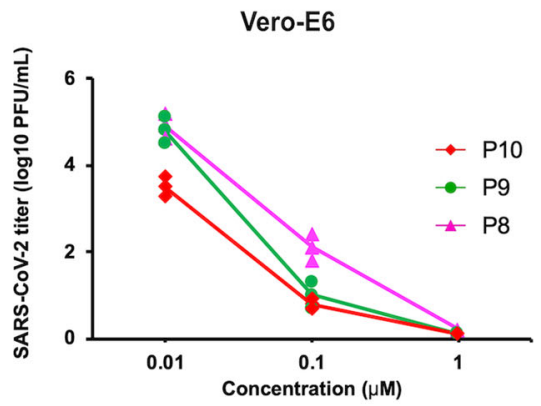

c)

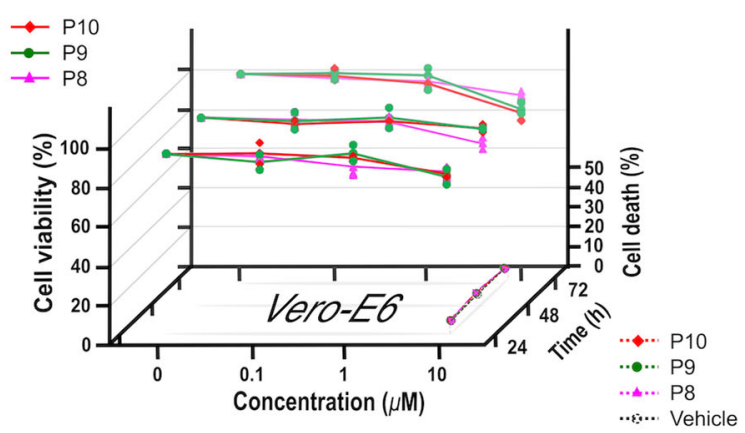

d)

Calu-3

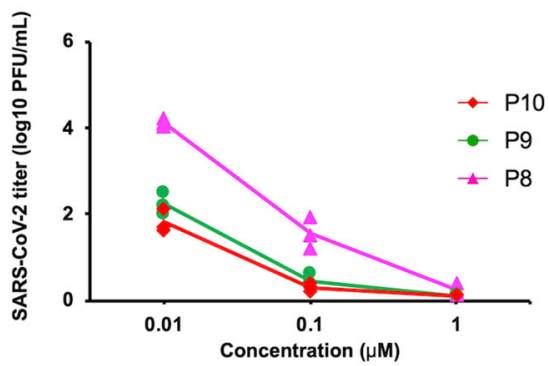

e)

Calu-3

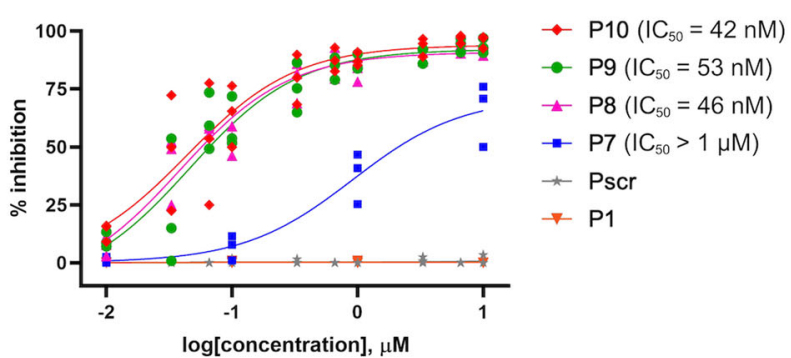

f)

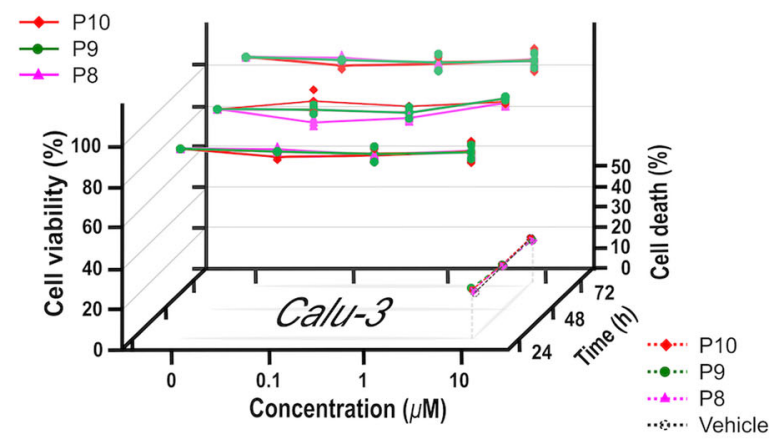

Fig. 3 Peptide-mimics of hACE2 show high anti-infective efficacy and are devoid of cell toxicity. a Percent inhibition of SARS-Cov-2 replication. Vero-E6 cells were infected with SARS-CoV-2/PSL2020 P\#2 stock at a multiplicity of infection (MOI) of 0.1 in the presence of 10 peptides (P1 to P8, P1scr, and Ppen as controls) at $10 \mu \mathrm{M}$ for $2 \mathrm{~h}$. Then, the virus was removed, and cultures were washed, incubated for $48 \mathrm{~h}$, before supernatant was collected to measure virus replication by ELISA. Data are combined from 3 to 6 independent experiments and expressed as compared to untreated SARS CoV-2infected Vero-E6 cells. b SARS-CoV-2 titer reduction in Vero-E6. Cells were infected with SARS-CoV-2/PSL2020 P\#2 stock in triplicate at a multiplicity of infection (MOI) of 0.1 in the presence of different concentrations (from 0.01 to $10 \mu \mathrm{M}$ ) of peptides P8, P9, and P10 for $2 \mathrm{~h}$. Then the virus was removed, and cultures were washed and incubated for $72 \mathrm{~h}$ to measure virus production by plaque assay. c Cell cytotoxicity in Vero-E6 cells. Cell viability was measured by MTT assays after treatment with vehicle $0,0.1,1$, or $10 \mu \mathrm{M}$ of $\mathbf{P 8}$, $\mathbf{P 9}$, or $\mathbf{P 1 0}$ for 24,48 , or $72 \mathrm{~h}$. Cell death was measured by flow cytometry using annexin-V-APC and PI staining in cells treated with vehicle or $10 \mu \mathrm{M} \mathbf{P 8}, \mathbf{P 9}$, or $\mathbf{P 1 0}$ for 24,48 , or $72 \mathrm{~h}$. The plots represent the means ( \pm SD) of three independent experiments. d SARS-CoV-2 titer reduction in Calu-3. Cells were infected with SARS-CoV-2/PSL2020 P\#2 stock in triplicate at a multiplicity of infection (MOI) of 0.3 in the presence of different concentrations (from 0.01 to $10 \mu \mathrm{M}$ ) of peptides P8, P9, and P10 for $2 \mathrm{~h}$, after which the virus was removed, and cultures were washed in, incubated for $72 \mathrm{~h}$ to measure virus production by plaque assay. e Dose-inhibition curve in Calu-3. Cells were infected with SARS-CoV-2/PSL2020 P\#2 stock at respectively a multiplicity of infection (MOI) of 0.3 in the presence of six different concentrations (from 0.01 to $10 \mu \mathrm{M}$ ) of peptides P1, P1 scr, P7, P8, P9, P10, for $2 \mathrm{~h}$. Then the virus was removed, and cultures were washed in, incubated for $48 \mathrm{~h}$, before supernatant was collected to measure virus replication by ELISA. Data are combined from 3 to 6 independent experiments and expressed as percent of inhibition compared to untreated SARS CoV-2-infected Vero-E6 cells. Data fitted in the sigmoidal dose-response curve represent the means ( \pm SD) of at least three independent experiments and are expressed as percent of inhibition compared to untreated SARS CoV-2-infected Vero-E6 cells. $\mathbf{f}$ Cell cytotoxicity in Calu-3 cells. Cell viability was measured by MTT assays after treatment with vehicle 0, 0.1, 1, or $10 \mu \mathrm{M}$ of $\mathbf{P 8}, \mathbf{P 9}$, or P10 for 24,48 , or $72 \mathrm{~h}$. Cell death was measured by flow cytometry using annexin-V-APC and PI staining in cells treated with vehicle or $10 \mu \mathrm{M} \mathbf{P 8}, \mathbf{P 9}$, or P10 for 24,48 , or $72 \mathrm{~h}$. The plots represent the means $( \pm S D)$ of three independent experiments.

\section{Methods}

\section{General chemistry}

Peptides syntheses. Peptides were produced manually, synthesized from Fmocprotected amino acids utilizing standard solid-phase peptide synthesis methods. Solid-phase peptide syntheses were performed in polypropylene Torviq syringes
(10 or $20 \mathrm{~mL}$ ) fitted with a polyethylene porous disk at the bottom and closed with an appropriate piston. Solvent and soluble reagents were removed through backand-forth movements. The appropriate protected amino acids were sequentially coupled using PyOxim/Oxyma as coupling reagents. The peptides were cleaved from the rink amide resin with classical cleavage cocktail trifluoroacetic acid/ 
a)

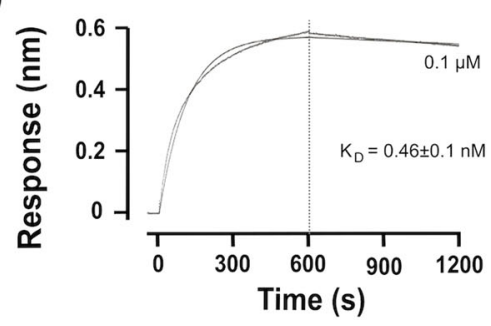

b)

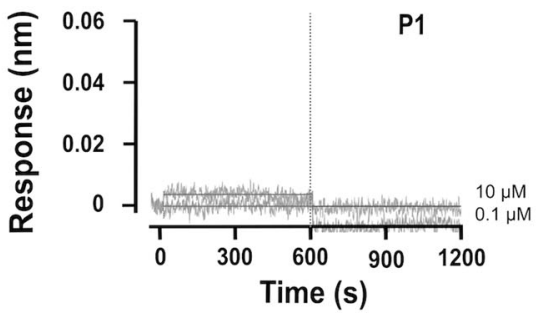

c)

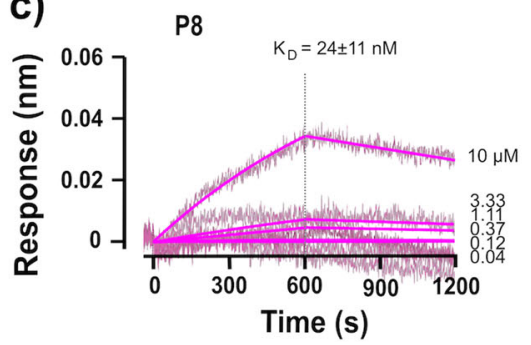

d)

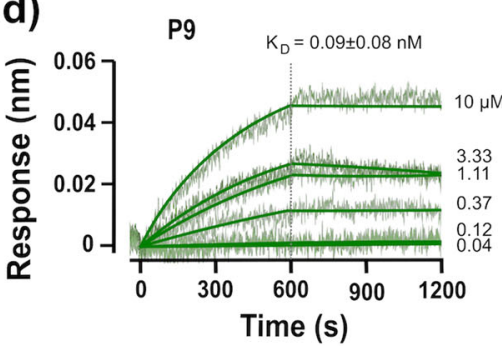

e)

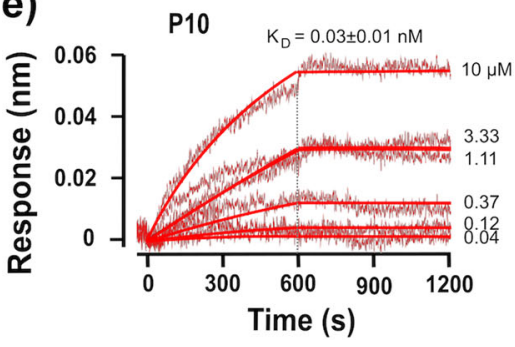

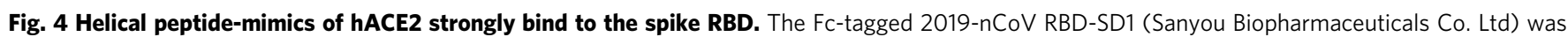
immobilized to an anti-human capture (AHC) sensortip (FortéBio) using an Octet RED96e system (FortéBio). The sensortip was then dipped into a $0.1 \mu \mathrm{M}$ solution of hACE2 (Sanyou Biopharmaceuticals Co. Ltd, His Tag), b 0.1 or $10 \mu \mathrm{M}$ solution of P1, or a range of concentrations (1 in 3 dilutions starting from $10 \mu \mathrm{M}$, i.e., $41 \mathrm{nM}, 123 \mathrm{nM}, 370 \mathrm{nM}, 1.1 \mu \mathrm{M}, 3.3 \mu \mathrm{M}$, and $10 \mu \mathrm{M})$ for $\mathbf{c} \mathbf{P 8}$, d P9, and e P10 to measure association rates before being dipped into a well containing only running buffer to measure dissociation rates. Data were reference subtracted and fitted to a 1:1 binding model using Octet Data Analysis Software v10.0 (FortéBio). All figures are representative of at least two independent experiments. $K_{D}$ values were calculated from Supplementary Table 6.

triisopropyl silane/water (95:2.5:2.5). The crude products were purified using preparative scale high-performance liquid chromatography (HPLC). The final products were characterized by analytical liquid chromatography (LC)-mass spectrometry (MS). All tested compounds were trifluoroacetic acid salts and were at least $95 \%$ pure. The relevant peptides after $\mathrm{CD}$ spectra analyses were selected and produced by Genecust France on $20 \mathrm{mg}$ scale.

Purification. Preparative scale purification of peptides was performed by reverse phase HPLC on a Waters system consisting of a quaternary gradient module (Water 2535) and a dual wavelength UV/visible absorbance detector (Waters 2489), piloted by Empower Pro 3 software using the following columns: preparative Macherey-Nagel column (Nucleodur HTec, C18, $250 \mathrm{~mm} \times 16 \mathrm{~mm}$ internal diameter, $5 \mu \mathrm{m}, 110 \AA$ ) and preparative Higgins analytical column (Proto 200, C18, $150 \mathrm{~mm} \times 20 \mathrm{~mm}$ i.d., $5 \mu \mathrm{m}, 200 \AA$ ) at a flow rate of $14 \mathrm{~mL} \mathrm{~min}{ }^{-1}$ and $20 \mathrm{~mL} \mathrm{~min}^{-1}$, respectively. Small-scale crudes $(<30 \mathrm{mg})$ were purified using semipreparative Ace column (Ace 5, C18, $250 \mathrm{~mm} \times 10 \mathrm{~mm}$ i.d., $5 \mu \mathrm{m}, 300 \AA$ ) at a flow rate of $5 \mathrm{~mL} \mathrm{~min}^{-1}$. Purification gradients were chosen to get a ramp of $\sim 1 \%$ solution B per minute in the interest area, and UV detection was done at 220 and $280 \mathrm{~nm}$. Peptide fractions from purification were analyzed by LC-MS (method A or B depending on retention time) or by analytical HPLC on a Dionex system consisting of an automated LC system (Ultimate 3000) equipped with an autosampler, a pump block composed of two ternary gradient pumps, and a dual wavelength detector, piloted by Chromeleon software. All LC-MS or HPLC analyses were performed on $\mathrm{C} 18$ columns. The pure fractions were gathered according to their purity and then freeze-dried using an Alpha 2/4 freeze-dryer from Bioblock Scientific to get the expected peptide as a white powder. Final peptide purity $(>95 \%)$ of the corresponding pooled fractions was checked by LC-MS using method A (outlined below).

Analytics. Two methods were conducted for LC-MS analysis. Method A. Analytical HPLC was conducted on a X-Select CSH C18 XP column $(30 \mathrm{~mm} \times 4.6 \mathrm{~mm}$ i. d., $2.5 \mu \mathrm{m}$ ), eluting with $0.1 \%$ formic acid in water (solvent $\mathrm{A}$ ) and $0.1 \%$ formic acid in acetonitrile (solvent $\mathrm{B}$ ), using the following elution gradient: $0-3.2 \mathrm{~min}$, $0-50 \% \mathrm{~B} ; 3.2-4 \mathrm{~min}, 100 \% \mathrm{~B}$. Flow rate was $1.8 \mathrm{~mL} \mathrm{~min}^{-1}$ at $40^{\circ} \mathrm{C}$. The mass spectra were recorded on a Waters $\mathrm{ZQ}$ mass spectrometer using electrospray positive ionization [ES + to give $(\mathrm{MH})+$ molecular ions] or electrospray negative ionization [ES-to give $(\mathrm{MH})$ - molecular ions] modes. The cone voltage was $20 \mathrm{~V}$. Method B. Analytical HPLC was conducted on a X-Select CSH C18 XP column ( $30 \mathrm{~mm} \times 4.6 \mathrm{~mm}$ i.d., $2.5 \mu \mathrm{m}$ ), eluting with $0.1 \%$ formic acid in water (solvent A) and $0.1 \%$ formic acid in acetonitrile (solvent $\mathrm{B}$ ), using the following elution gradient: $0-3.2 \mathrm{~min}, 5-100 \% \mathrm{~B} ; 3.2-4 \mathrm{~min}, 100 \%$ B. Flow rate was $1.8 \mathrm{~mL} \mathrm{~min}^{-1}$ at $40^{\circ} \mathrm{C}$. The mass spectra were recorded on a Waters ZQ mass spectrometer using electrospray positive ionization [ES + to give $(\mathrm{MH})+$ molecular ions] or electrospray negative ionization [ES - to give $(\mathrm{MH})-$ molecular ions] modes. The cone voltage was $20 \mathrm{~V}$.
CD spectroscopy. CD spectra were recorded on a Jasco J-815 CD spectropolarimeter equipped with a Peltier temperature controller. Data were obtained at $25^{\circ} \mathrm{C}$ over a wavelength range between $185 \mathrm{~nm}$ and $270 \mathrm{~nm}$, using a wavelength interval of $0.2 \mathrm{~nm}$ and a scan rate of $20 \mathrm{~nm} \mathrm{~min}{ }^{-1}$. Peptide samples were prepared at a concentration of $60 \mu \mathrm{M}$ in $50 \mathrm{mM}$ sodium phosphate buffer, $\mathrm{pH} 7.4$, in a quartz cell of $1 \mathrm{~mm}$ path length. $\mathrm{CD}$ experiments were processed and plotted with $\mathrm{R}$ program. CD spectra were analyzed using DICHROWEB web server and CDSSTR deconvolution algorithm ${ }^{24}$.

\section{Anti-infectivity study on Calu-3 and Vero-E6 cells}

Cells and virus preparation. Calu-3 (ATCC HTB55) and Vero-E6 (ATCC CRL1586) cells were purchased from the American Type Culture Collection and routinely checked for mycoplasma contamination ${ }^{26}$. Cells were cultured in Dulbecco's Modified Eagle Medium (DMEM) supplemented with non-essential amino acids, penicillin-streptomycin, and $10 \% \mathrm{v} / \mathrm{v}$ fetal bovine serum.

The SARS-CoV-2 clinical isolate was obtained from BAL of a symptomatic infected patient (\#SARS-CoV-2/PSL2020, available at Pitié-Salpêtrière hospital, Paris (France)). The patient recruited for virus isolation and culture was in intensive care unit in the Pitié Salpêtrière hospital. The patient underwent a bronchoalveolar lavage for clinical purpose (seeking for a bacterial pulmonary infection). The protocol was approved by our institution's ethics committee (Immuno-COVID-REA, CER-Sorbonne Université, no. CER-SU-2020-31). BAL $(0.5 \mathrm{~mL})$ was mixed with an equal volume of DMEM without FBS, supplemented with $25 \mathrm{mM}$ Hepes, double concentration of penicillin-streptomycin and miconazole (Sigma), and added to $80 \%$ confluent Vero-E6 cells monolayer seeded into a $25-\mathrm{cm}^{2}$ tissue culture flask. After $1 \mathrm{~h}$ adsorption at $37^{\circ} \mathrm{C}, 3 \mathrm{~mL}$ of infectious media (DMEM supplemented with $2 \%$ FBS, penicillin-streptomycin and miconazole) were added. Twenty-four hours post-infection another $2 \mathrm{~mL}$ of infectious media were added. Five days post-infection, supernatants were collected, aliquoted, and stored at $-80^{\circ} \mathrm{C}(\mathrm{P} \# 1)$. For secondary virus stock, Vero-E6 cells seeded into $25 \mathrm{~cm}^{2}$ tissue culture flasks were infected with $0.5 \mathrm{~mL}$ of P\#1 stored aliquot, and cell-culture supernatant were collected $48 \mathrm{~h}$ post-infection and stored at $-80^{\circ} \mathrm{C}(\mathrm{P} \# 2)$. Infectious viral particles were measured by a standard plaque assay previously described ${ }^{25}$ with fixation of cells $72 \mathrm{~h}$ post infection. Accordingly, the viral titer of SARS-CoV-2/PSL2020 P\#2 stock was about 5.3 $10^{5} \mathrm{PFU} \mathrm{mL}^{-1}$.

Viral neutralization. Vero-E6 or Calu-3 $\left(1 \times 10^{5}\right.$ cells $\left.\mathrm{mL}^{-1}\right)$ were seeded into 24 wells plates in infectious media and treated with different concentrations of the peptides (from 0.1 to $10 \mu \mathrm{M}$ ). After $30 \mathrm{~min}$ at room temperature, cells were infected with 0.1 multiplicity of infection (MOI) (Vero-E6) or 0.3 MOI (Calu-3) of SRASCoV-2 (SARS-CoV-2/PSL2020 P\#2 stock) in infectious media. Cell supernatants were collected at $48 \mathrm{~h}$ post-infection for enzyme-linked immunosorbent assay (ELISA) assay using a SARS-CoV-2 (2019-nCoV) nucleoprotein ELISA kit (Sino biological), according the manufacturer's instructions, and standard plaque assay ${ }^{26}$. 
Inhibition of infection was calculated comparing viral concentration in each case with that of untreated SARS- CoV-2-infected cells.

Toxicity study on Calu-3 and Vero-E6 cells. Cell cytotoxicity was measured using MTT assays and fluorescent activated cell sorting (FACS). Calu-3 and VeroE6 were seeded in 96-well (for MTT assays) or 24-well (for FACS) plates and let adhere. When $\sim 40 \%$ confluence was reached, the wells were washed, and new medium containing vehicle (water, $5 \%$ final volume) or different concentrations of the indicated peptide was added. MTT $(2 \mathrm{mM})$ was added to each well after $24 \mathrm{~h}$, $48 \mathrm{~h}$, or $72 \mathrm{~h}$ of treatment and incubated $4 \mathrm{~h}$ at $37^{\circ} \mathrm{C}$. Supernatants were then discarded and formazan salts were dissolved in DMSO to read plate absorbance at $570 \mathrm{nM}$. Absorbance in each well was normalized with those treated with vehicle. For FACS analyses, cells were harvested from the wells with the help of accutase, pelleted, and stained with Annexin-V-APC $\left(0.1 \mu \mathrm{g} \mathrm{mL}^{-1}\right)$ and propidium iodide $\left(0.5 \mu \mathrm{g} \mathrm{mL}^{-1}\right)$ in annexin binding buffer $(10 \mathrm{mM}$ Hepes $\mathrm{pH} 7.4,140 \mathrm{mM} \mathrm{NaCl}$, and $2.5 \mathrm{mM} \mathrm{CaCl}$ ). Cells were sorted in a FACScalibur flow cytometer and data was analyzed using FlowJo 10.0 software, considering cell death as the sum of Ann- $\mathrm{V}^{+} / \mathrm{PI}^{-}$and Ann- $\mathrm{V}^{+} / \mathrm{PI}^{+}$events.

Biolayer Interferometry experiments. Fc-tagged 2019-nCoV RBD-SD1 (Sanyou Biopharmaceuticals Co. Ltd) was immobilized to an anti-human capture sensortip (FortéBio) using an Octet RED96e (FortéBio). The sensortip was then dipped into $100 \mathrm{nM}$ hACE2 (Sanyou Biopharmaceuticals Co. Ltd, His Tag) or $1 \mu \mathrm{M}$ of any tested peptide to measure association before being dipped into a well containing only running buffer composed of DPBS (Potassium Chloride $2.6 \mathrm{mM}$, Potassium Phosphate monobasic $1.5 \mathrm{mM}$, Sodium Chloride $138 \mathrm{mM}$, Sodium Phosphate dibasic $8 \mathrm{mM}$ ), $0.05 \%$ Tween 20 and $0.5 \%$ bovine serum albumin to measure dissociation. (Extended conditions, and suppliers in Supplementary Note 1).

Statistics and reproducibility. All conditions in the viral neutralization and cell cytotoxicity experiments were tested in triplicate. Data from viral neutralization assays was analyzed using nonlinear regression, fitting sigmoidal curves used to calculate the $\mathrm{IC}_{50}$ values with Graphpad Prism 8.0.1. For BLI experiments, data were reference subtracted and fit to a 1:1 binding model using Octet Data Analysis Software v10.0 (FortéBio). $K_{\mathrm{D}}$ values were extracted from 3 independent determinations (P9 and P10) validated by a statistical coefficient determination $\left(R^{2}\right)>0.95$.

Reporting summary. Further information on research design is available in the Nature Research Reporting Summary linked to this article.

\section{Data availability}

The complex between hACE2 and the surface spike protein of SARS-CoV-2 data used for peptide design is available in RCSB PDB with the identifier doi: $10.2210 / \mathrm{pdb} 6 \mathrm{M} 0 \mathrm{~J} / \mathrm{pdb}^{14}$. The randomly selected RBD amino-acid sequences from China (QOH25833, QIG55857, QHR63290, QHR63250, QJG65957, QJG65956, and QJG65951), USA (QIV65044, QJD23847, QJU11481, QJD24531, QJD25193, QJD25529, and QJA17180), and France (QJT73034, QJT73010, QJT72902, QJT72806, QJT72794, QJT72722, QJT72710, QJT72626, QJT72614, and QJT72554), used for sequence alignment are available in the Genebank database at ncbi.nlm.nih.gov. The amino acid sequence of the Fc-tagged 2019nCoV RBD-SD1 was provided by Sanyou bio with purchase (sanyoubio.com/EN/2019nCoV.php) and is depicted in Supplementary Fig. 1a. The datasets generated and/or analyzed during the current study are in the manuscript and the raw data are available from the corresponding author on reasonable and justified request.

\section{Code availability}

The predictive data supporting our work was generated using publicly available software. For sequence alignment, the Weblogo software (available at https://weblogo.berkeley. edu) and Clustal Omega (available at https://ebi.ac.uk/Tools/msa/clustalo/) were used. The Agadir helical content predictor is available at https://agadir.crg.es. Peptide antigenicity can be determined at https://imed.med.ucm.es/Tools/antigenic.pl. Online analysis for peptide CD spectra and using DichroWeb server and the CDSSTR algorithm is available at https://dichroweb.cryst.bbk.ac.uk. The HELIQUEST web server used to calculate peptide hydrophobicity and hydrophobic moment is available at https:// heliquest.ipmc.cnrs.fr.

Received: 31 July 2020; Accepted: 20 January 2021; Published online: 12 February 2021

\section{References}

1. WHO. Coronavirus disease (COVID-19) Weekly Operational Update - 20 November 2020. https://www.who.int/emergencies/diseases/novelcoronavirus-2019/situation-reports (2020).
2. Wang, C., Horby, P. W., Hayden, F. G. \& Gao, G. F. A novel coronavirus outbreak of global health concern. Lancet 395, 470-473 (2020).

3. Zhu, N. et al. A novel coronavirus from patients with pneumonia in China, 2019. N. Engl. J. Med. 382, 727-733 (2020).

4. ANSM. COVID-19 - Essais cliniques en cours https://www.ansm.sante.fr/ Activites/Essais-cliniques/COVID-19-Essais-cliniques-en-cours/(offset)/0 (2020).

5. Kupferschmidt, K. These drugs don't target the coronavirus-they target us. Science (80-.) https://doi.org/10.1126/science.abc0405 (2020).

6. Shang, J. et al. Structural basis of receptor recognition by SARS-CoV-2. Nature 581, 221-224 (2020).

7. Yan, R. et al. Structural basis for the recognition of SARS-CoV-2 by full-length human ACE2. Science (80-) 367, 1444-1448 (2020).

8. Hoffmann, M. et al. SARS-CoV-2 cell entry depends on ACE2 and TMPRSS2 and is blocked by a clinically proven protease inhibitor. Cell 181, 271-280.e8 (2020).

9. Monteil, V. et al. Inhibition of SARS-CoV-2 infections in engineered human tissues using clinical-grade soluble human ACE2. Cell 181, 905-913.e7 (2020).

10. Romano, M., Ruggiero, A., Squeglia, F. \& Berisio, R. Engineered stable miniprotein plug SARS-Cov-2 spikes. Preprint at https://doi.org/10.1101/ 2020.04.29.067728 (2020).

11. Ling, R. et al. In silico design of antiviral peptides targeting the spike protein of SARS-CoV-2. Peptides 130, 170328 (2020).

12. Han, Y. \& Král, P. Computational design of ACE2-based peptide inhibitors of SARS-CoV-2. ACS Nano 14, 5143-5147 (2020).

13. Zhang, G. et al. Investigation of ACE2 N-terminal fragments binding to SARSCoV-2 Spike RBD. Preprint at https://doi.org/10.1101/2020.03.19.999318 (2020).

14. Lan, J. et al. Structure of the SARS-CoV-2 spike receptor-binding domain bound to the ACE2 receptor. Nature 581, 215-220 (2020).

15. Chakrabartty, A., Kortemme, T. \& Baldwin, R. L. Helix propensities of the amino acids measured in alanine-based peptides without helix-stabilizing side-chain interactions. Protein Sci. 3, 843-852 (1994).

16. Klein, M. A. Stabilized helical peptides: a strategy to target protein-protein interactions. ACS Med. Chem. Lett. 5, 838-9 (2014).

17. Chapman, R. N., Dimartino, G. \& Arora, P. S. A highly stable short alphahelix constrained by a main-chain hydrogen-bond surrogate. J. Am. Chem. Soc. 126, 12252-3 (2004).

18. Muñoz, V. \& Serrano, L. Elucidating the folding problem of helical peptides using empirical parameters. Nat. Struct. Mol. Biol. 1, 399-409 (1994).

19. Muñoz, V. \& Serrano, L. Elucidating the folding problem of helical peptides using empirical parameters. II. Helix macrodipole effects and rational modification of the helical content of natural peptides. J. Mol. Biol. 245, 275-96 (1995).

20. Kolaskar, A. S. \& Tongaonkar, P. C. A semi-empirical method for prediction of antigenic determinants on protein antigens. FEBS Lett. 276, 172-4 (1990).

21. Harper, E. T. \& Rose, G. D. Helix stop signals in proteins and peptides: the capping box. Biochemistry 32, 7605-9 (1993).

22. Karoyan, P. et al. In Targets in Heterocyclic Systems. p. 216-273 (Societa chimica italiana, 2004).

23. Cordes, F. S., Bright, J. N. \& Sansom, M. S. P. Proline-induced distortions of transmembrane helices. J. Mol. Biol. 323, 951-60 (2002).

24. Okumura, H. S., Philmus, B., Portmann, C. \& Hemscheidt, T. K. Homotyrosine-containing cyanopeptolins 880 and 960 and anabaenopeptins 908 and 915 from Planktothrix agardhii CYA 126/8. J. Nat. Prod. 72, 172-6 (2009).

25. Whitmore, L. \& Wallace, B. A. DICHROWEB, an online server for protein secondary structure analyses from circular dichroism spectroscopic data. Nucleic Acids Res. 32, W668-73 (2004).

26. Mendoza, E. J., Manguiat, K., Wood, H. \& Drebot, M. Two detailed plaque assay protocols for the quantification of infectious SARS-CoV-2. Curr. Protoc. Microbiol. 57, ecpmc105 (2020).

27. Sheahan, T. P. et al. An orally bioavailable broad-spectrum antiviral inhibits SARS-CoV-2 in human airway epithelial cell cultures and multiple coronaviruses in mice. Sci. Transl. Med. 12, eabb5883 (2020).

28. Zhu, Y., Chidekel, A. \& Shaffer, T. H. Cultured human airway epithelial cells (CALU-3): a model of human respiratory function, structure, and inflammatory responses. Crit. Care Res. Pract. 2010, 394578 (2010).

29. $\mathrm{Chu}, \mathrm{H}$. et al. Comparative tropism, replication kinetics, and cell damage profiling of SARS-CoV-2 and SARS-CoV with implications for clinical manifestations, transmissibility, and laboratory studies of COVID-19: an observational study. Lancet Microbe 1, e14-e23 (2020).

30. Wrapp, D. et al. Cryo-EM structure of the 2019-nCoV spike in the prefusion conformation. Science 367, 1260-1263 (2020).

31. Cao, L. et al. De novo design of picomolar SARS-CoV-2 miniprotein inhibitors. Science 370, 426-431 (2020).

32. Pinto, D. et al. Cross-neutralization of SARS-CoV-2 by a human monoclonal SARS-CoV antibody. Nature 583, 290-295 (2020). 
33. Fosgerau, K. \& Hoffmann, T. Peptide therapeutics: current status and future directions. Drug Discov. Today 20, 122-8 (2015).

34. Lau, J. L. \& Dunn, M. K. Therapeutic peptides: Historical perspectives, current development trends, and future directions. Bioorg. Med. Chem. 26, 2700-2707 (2018).

35. Vlieghe, P., Lisowski, V., Martinez, J. \& Khrestchatisky, M. Synthetic therapeutic peptides: science and market. Drug Discov. Today 15, 40-56 (2010).

36. Uhlig, $\mathrm{T}$. et al. The emergence of peptides in the pharmaceutical business: from exploration to exploitation. EuPA Open Proteom. 4, 58-69 (2014).

37. Lax, R. The Future Of Peptide Development In The Pharmaceutical Industry. https://www.semanticscholar.org/paper/The-Future-of-PeptideDevelopment-in-the-Industry-Lax/91e7e891ef217610b8f1d04a6d920a20c74e4091 (2010).

38. Gupta, V. Glucagon-like peptide-1 analogues: an overview. Indian. J. Endocrinol. Metab. 17, 413 (2013).

39. Zhou, N. E., Kay, C. M., Sykes, B. D. \& Hodges, R. S. A single-stranded amphipathic alpha-helix in aqueous solution: design, structural characterization, and its application for determining alpha-helical propensities of amino acids. Biochemistry 32, 6190-7 (1993).

\section{Acknowledgements}

This work was supported by private funds (P.K.), SATT-Lutech, Kaybiotix (LGM PhD grant), and French Research Ministry (EO PhD grant). P.K. is grateful to SATT-Lutech team supporting this project, to Fabrice Viviani and Akanksha Gangar from Oncodesign for their unwavering support. The authors thank David Boutolleau from the Virology Department, Pitié-Salpêtrière, AP-HP, where was diagnosed the BAL SARS-COV-2 infection. Philippe Karoyan dedicates this work to Gérard Chassaing on the occasion of his 75 th birthday. "There are no borders in science. The only limit is our imagination."

\section{Author contributions}

P.K. conceived and supervised this project, designed and synthesized the peptides, designed the experiments, interpreted the data, and wrote the draft and the discussion of the manuscript. P.K. and O.L. wrote the manuscript. P.K. and O.L. performed the molecular modeling study. A.D. contributed to the molecular modeling study.

O.L. performed the CD structural studies. V.V. performed the cell inhibition assays. P.G. performed the binding experiments. E.O. performed the peptides LC-MS analyses. L.G.M. performed the cell toxicity experiments. A.G. and C.E.L. recruited the patient and provided BAL sample

\section{Competing interests}

The authors declare the following competing financial interest(s): The patent application EP20305449.9 included results from this paper.

\section{Additional information}

Supplementary information The online version contains supplementary material available at https://doi.org/10.1038/s42003-021-01736-8.

Correspondence and requests for materials should be addressed to P.K.

Reprints and permission information is available at http://www.nature.com/reprints

Publisher's note Springer Nature remains neutral with regard to jurisdictional claims in published maps and institutional affiliations.

(c) (i) adaptation, distribution and reproduction in any medium or format, as long as you give appropriate credit to the original author(s) and the source, provide a link to the Creative Commons license, and indicate if changes were made. The images or other third party material in this article are included in the article's Creative Commons license, unless indicated otherwise in a credit line to the material. If material is not included in the article's Creative Commons license and your intended use is not permitted by statutory regulation or exceeds the permitted use, you will need to obtain permission directly from the copyright holder. To view a copy of this license, visit http://creativecommons.org/ licenses/by/4.0/.

(C) The Author(s) 2021 